\title{
Questionário de Necessidade de Emoções (NAQ-S): Validade de Construto, Invariância e Fidedignidade
}

\author{
Gabriel Lins de Holanda Coelho - Cardiff University, Reino Unido \\ Gregory R. Maio - University of Bath, Reino Unido \\ Valdiney V. Gouveia - Universidade Federal da Paraíba, João Pessoa, Brasil \\ Lukas Jarmo Wolf - Cardiff University, Reino Unido \\ Renan Pereira Monteiro - Universidade Federal de Mato Grosso, Cuiabá, Brasil
}

\begin{abstract}
Resumo
Dois estudos $(N=457)$ examinaram as propriedades psicométricas da versão reduzida do Questionário de Necessidade de Emoções (Need for Affect Questionnaire: NAQ-S) no contexto brasileiro. No primeiro estudo, uma análise de componentes principais indicou uma estrutura bifatorial, com cinco itens cada: aproximação $(\alpha=0,70)$ e evitação $(\alpha=0,75)$. Confirmou-se essa estrutura no segundo estudo (GFI $=0,92 ; \mathrm{CFI}=0,90)$, mostrando-se invariante quanto ao sexo. Para verificar os correlatos do NAQ-S, utilizou-se, nos dois estudos, medidas que visam avaliar os valores humanos, a solidão e a necessidade de pertença, além dos cinco fatores da personalidade no segundo estudo. Os resultados demonstraram que uma maior necessidade de emoção foi associada com maior extroversão, maiores escores em valores de experimentação e interativos, e escores mais baixos de solidão. Os estudos apresentam suporte de validade do NAQ-S no Brasil, mostrando sua utilidade para fins de pesquisa.

Palavras-chave: necessidade de emoções, validação, parâmetros psicométricos
\end{abstract}

\section{Need for Affect Questionnaire (NAQ-S): Construct Validity, Invariance and Reliability}

\begin{abstract}
Two studies ( $\mathrm{N}=457$ ) examined the psychometric properties of the short version of the Need for Affect Questionnaire (NAQ$S)$ in the Brazilian context. In the first study, a principal components analysis indicated a two-factor structure, with five items each: approach $(\alpha=.70)$ and avoidance $(\alpha=.75)$. This structure was confirmed in the second study (GFI $=.92$; CFI $=.90)$, and it was invariant across gender. To verify the correlates of NAQ-S, we used measures to evaluate the human values, loneliness and need to belong in both studies, besides the five personality factors in the second study. The results showed that higher need for affect was associated with higher extraversion, higher scores in excitement and interactive values, and with lower loneliness scores. The studies support the validity of the NAQ-S in Brazil, showing its usefulness for research purposes.
\end{abstract}

Keywords: need for affect; validation; psychometric parameters

\section{Cuestionario de Necesidad de Emóciones (NAQ-S): Validez de Constructo, Invariancia y Fiabilidad}

\begin{abstract}
Resumen
Dos estudios $(\mathrm{N}=457)$ examinaron las propriedades psicométricas de la versión reducida del Cuestionario de Necesidad de Emociones (Need for Affect Questionnaire; NAQ-S) en el contexto brasileño. En el primer estudio, un análisis de componentes principales indicó una estructura bifactorial, con cinco ítems cada una: aproximación $(\alpha=.70)$ y evitación $(\alpha=.75)$. Se confirmó esta estructura en el segundo estudio ( $\mathrm{GFI}=.92 ; \mathrm{FCI}=.90)$, mostrándose invariable con relación al sexo. Para verificar las correlaciones del NAQ-S, fue utilizado en ambos estudios medidas que tienden a evaluar los valores humanos, la soledad y la necesidad de pertenencia, además de evaluarse en el segundo estudio los cinco factores de personalidad. Los resultados mostraron que una mayor necesidad de emoción, fue asociada con mayor extraversión, con resultados mayores en valores de experimentación e interactivos, y resultados más bajos de soledad. Los estudios presentan apoyo de validez del NAQ-S en Brasil, mostrando su utilidad para fines de investigación.

Palabras-clave: necesidad de emociones, validación, parámetros psicométricos.
\end{abstract}

\section{Introdução}

As emoções se configuram como um tema de estudo de diferentes áreas do conhecimento (e.g., antropologia, psicologia, neurociência; Dalgleish, Dunn, \& Mobbs, 2009; Lutz \& White, 1986). Especificamente no âmbito das diferenças individuais em aspectos emocionais, Maio e Esses (2001) apontam que as pesquisas têm se concentrado em capacidades como regulação, percepção e expressão das emoções. Não obstante, pouca atenção tem sido dada à necessidade de emoções (Need for Affect - NFA). Concretamente, a NFA descreve pessoas que buscam situações que eliciam emoções, que desfrutam de estímulos emocionais e usam as emoções em sua interação com o mundo (Raman, Chattopadhyay, \& Hoyer, 1995). Nessa direção, a NFA representa a motivação das pessoas para se aproximar ou evitar 
emoções ou situações que possam induzi-las (Maio \& Esses, 2001).

O componente motivacional que envolve a NFA é o aspecto que a diferencia de construtos correlatos que focam, por exemplo, em como as emoções são vivenciadas e/ou expressas (Appel, Gnambs, \& Maio, 2012). De acordo com Maio e Esses (2001), a conceituação da NFA perpassa por duas características. A primeira assume que as pessoas diferem quanto à busca por experiências emocionais, e a segunda se refere à possibilidade de responder às emoções de forma positiva ou negativa. Em razão disso, os autores indicam que o construto atua como uma espécie de atitude em relação à emoção.

Essa necessidade tem em conta em que medida as pessoas desejam compreender suas emoções e as dos outros, além de avaliar crenças sobre a utilidade das emoções para moldar decisões e comportamentos (Maio \& Esses, 2001). Tendo em vista tais aspectos, estudos têm sido realizados considerando a NFA para entender diferentes construtos. Por exemplo, Leone e Chirumbolo (2008) verificaram que evitar emoções se correlacionou positivamente com o endosso a políticas conservadoras (e.g., autoritarismo de direita, dominância social), enquanto correlações negativas foram observadas da aproximação com tais disposições conservadoras. Ainda no âmbito da Psicologia Política, Arceneaux e Wielen (2013), em estudo experimental que expôs as pessoas a informações negativas sobre partidos políticos, verificaram que aqueles com elevados escores em NFA e baixos em necessidade de cognição (Need for Cognition - NFC) avaliaram mais duramente o partido de oposição do que o seu próprio partido, além das informações negativas sobre o partido de oposição reforçarem positivamente as avaliações ao próprio partido. Os autores concluem que as pessoas com elevada NFA foram mais apegadas emocionalmente a seus partidos, aspecto que influenciou nos julgamentos.

Em outro estudo, Haddock, Maio, Arnold e Huskinson (2008) verificam que mensagens persuasivas de cunho emocional eliciam respostas mais positivas entre aqueles que pontuam alto em NFA. Portanto, Haddock et al (2008). apontam que pessoas pautadas na busca de emoções procuram informações de cunho emocional para formar suas atitudes, aspecto este importante de se ter em conta, por exemplo, na área da psicologia do consumidor (Raman et al., 1995). De fato, tal como apontam Cho e Workman (2014), algumas marcas utilizam em suas propagandas imagens que remetem a conteúdos de base emocional, algo que pode aumentar a intenção de compra dos consumidores, sobretudo daqueles que pontuam alto em NFA.

Reforçando o importante papel da NFA nos comportamentos, observa-se o estudo desenvolvido por Corwin, Cramer, Griffin e Brodsky (2012), onde os participantes foram solicitados a indicar a sentença (prisão perpétua ou pena de morte) que dariam a um indivíduo descrito como homicida. Aqueles que pontuaram mais no fator aproximação da NAQ se mostraram mais favoráveis a uma pena mais branda, isto é, à prisão perpétua.

A partir das evidências empíricas expostas, se constata que aproximar-se ou não de emoções tem repercussões concretas em comportamentos e julgamentos (Maio \& Esses, 2001), como, por exemplo, influenciando a avaliação de partidos políticos, decisões condenatórias ou consumo. Apesar da relevância de se estudar a NFA, as pesquisas sobre esse construto têm sido menos frequentes comparado com aqueles que consideram, por exemplo, inteligência emocional, alexitimia e afetos positivos e negativos, possivelmente devido à carência de medidas que operacionalizam o fenômeno (Maio \& Esses, 2001). Precisamente, esse aspecto levou Maio e Esses a propor o Questionário de Necessidade de Emoções (Need for Affect Questionnaire NAQ), que pode ser útil na exploração de uma série de problemas na regulação do comportamento relevante para a emoção. Considerando a importância de medir tal necessidade, este artigo tem como objetivo adaptar essa medida para o contexto brasileiro, considerando sua versão reduzida.

Questionário de Necessidade de Emocões, Versão Reduzida (Need for Affect Questionnaire, short form; NAQ-S)

A NAQ foi originalmente elaborada por Maio e Esses (2001), avaliando dois fatores: aproximação e evitação. Inicialmente, eles reuniram um conjunto de 88 itens, depois reduzidos a $60 \mathrm{com}$ a análise de juízes. Essa versão foi aplicada a 355 pessoas, que responderam os itens em escala de 7 pontos (-3 = Discordo fortemente a $3=$ Concordo fortemente). Os dados foram submetidos a diferentes análises fatoriais exploratórias, usando distintos métodos de retenção de fatores e rotação, sendo consistente a estrutura bifatorial. Pautados nesses resultados, os autores selecionaram 29 itens com saturações acima de 0,30; 15 para o fator Aproximação e 14 para Evitação. Não obstante, verificaram que três deles possuíam baixa correlação inter-item, passando a considerar uma versão com 26 itens, 13 para cada fator. Essa versão foi testada, sendo a solução bifatorial superior a unifatorial (e.g., SMSR = 0,07 e RMSEA = 0,06). 
Posteriormente, Maio e Esses (2001) procuraram conhecer evidências de validades convergente e discriminante da NAQ com construtos que avaliam domínios afetivos, estilos cognitivos e ativação e inibição comportamental. Além disso, verificaram que, dos cinco grandes fatores da personalidade, quatro se correlacionaram com a pontuação agregada da NAQ (aproximação - evitação): Extroversão $(r=0,36, p$ $<0,05)$, Abertura $(r=0,29, p<0,05)$, Amabilidade $(r=0,24, p<0,05)$ e Neuroticismo $(r=-0,22, p<$ $0,05)$. Por fim, eles avaliaram a validade preditiva da NAQ, que se correlacionou positivamente com atitudes extremistas e a disposição para assistir filmes de caráter emotivo, por exemplo.

Apesar de existirem evidências de adequação psicométrica da NAQ com 26 itens (Appel, 2008; Leone \& Presaghi, 2007), um problema inerente dessa versão é o seu tamanho; é particularmente problemático se for levado em conta sua aplicação em conjunto com outros instrumentos. Nessa direção, nos últimos anos tem-se verificado a importância de instrumentos reduzidos, uma vez que as circunstâncias da coleta de dados não são comumente as ideais, podendo ocasionar problemas como fadiga ou tédio (Gosling, Rentfrow, \& Swann Jr, 2003).

Sendo assim, Appel et al. (2012) propuseram elaborar uma versão reduzida do instrumento. No caso, preservando elementos-chave da conceituação da necessidade de emoção, estes autores consideraram as cargas fatoriais mais elevadas nos estudos de Maio e Esses (2001) e Appel (2008), chegando a uma versão com dez itens igualmente distribuídos nos dois fatores dessa medida. Posteriormente, considerando quatro amostras independentes, Appel et al. (2012) procuraram conhecer evidências de validade fatorial dessa medida, checando sua invariância quanto ao sexo, idade e nível educacional dos participantes, a correlação das pontuações de seus fatores com aquelas da necessidade de cognição e dos cinco grandes fatores da personalidade, além da consistência interna. Eles observaram que o modelo bifatorial da NAQ-S apresentou indicadores de ajuste aceitáveis (e.g., CFI > $0,90$, RMSEA $\leq 0,05)$. O escore agregado da NAQ-S se correlacionou com aquele de necessidade de cognição $(r=0,17$ para versão reduzida, e 0,15 para a original) e os cinco grandes fatores de personalidade (versões reduzida e original, respectivamente): Abertura $(r=0,31$ para ambas), Conscienciosidade $(r=$ $0,19$ e 0,25$)$, Extroversão $(r=0,42$ e 0,46$)$, Amabilidade $(r=0,39$ e 0,41$)$ e Neuroticismo $(r=0,16$ e 0,19$)$.
Quanto à consistência interna (alfa de Cronbach), os coeficientes variaram entre 0,72 e 0,82 .

Appel, Gnambs e Maio (2012) também consideraram os dois fatores específicos da NAQ-S, tendo os resultados de correlação com os cinco fatores de personalidade igualmente apresentado similaridades entre suas versões original e reduzida; apenas em relação ao fator Conscienciosidade as correlações foram algo mais discrepantes $(r=0,29$ na versão original, e 0,19 na reduzida). Não obstante, ressalta-se que no estudo de Maio e Esses (2001) não foram encontradas correlações entre os fatores da NAQ e o fator Conscienciosidade.

A partir dos resultados descritos por Appel et al. (2012), percebe-se a possibilidade de utilização da versão reduzida da NAQ, uma vez que a estrutura bifatorial mostrou-se adequada e os correlatos, comparando-os à versão original, foram similares. Desse modo, justifica-se tê-la em conta, procurando adaptá-la à realidade brasileira. Para isso, foram realizados dois estudos, objetivando reunir evidências de validade fatorial e consistência interna da medida, bem como correlatos e invariância fatorial quanto ao sexo.

No que tange aos correlatos, foram avaliadas as associações da NAQ-S com os cinco fatores da personalidade, buscando comparar o padrão de relações com os estudos de Maio e Esses (2001) e Appel et al. (2012). Ademais, buscou-se verificar em que medida a NAQ-S se correlaciona com os valores humanos, sobretudo por estes representarem cognitivamente as necessidades (Gouveia, Milfont, \& Guerra, 2014). Baseado na teoria funcionalista dos valores (Gouveia, 2013), é possível pensar que pessoas pautadas por buscar emoções, descobrir e apreciar novos estímulos (Valores de experimentação) e que buscam às interações sociais e valorizam experiências afetivas (Valores interativos), apresentam maiores níveis de NFA.

Por fim, foram consideradas as correlações da NAQ-S com necessidade de pertença e solidão. Estes dois últimos construtos compartilham a percepção subjetiva de conexão com os demais, onde o não atendimento das necessidades de pertença pode contribuir para o surgimento da solidão (Baumeister \& Leary, 1995; Mellor, Stokes, Firth, Hayashi, \& Cummins, 2008). Especificamente, Baumeister e Leary apontam que formar vínculos sociais pode resultar em respostas emocionais, onde o aumento em pertencimento pode estar relacionado a afetos positivos, ao passo que uma privação de interação com os demais pode levar a afetos negativos. Logo, os afetos positivos que surgem em função da vinculação com outras pessoas podem 
reforçar a busca por aproximar-se das emoções e de situações que possam induzi-las. Por outro lado, para manterem-se longe dos afetos negativos que permeiam a solidão, as pessoas buscariam afastar-se das emoções.

Considerando o previamente exposto, buscou-se avaliar a estrutura fatorial da NAQ-S, verificando se essa é invariante quanto ao sexo. Além disso, buscou-se conhecer o padrão de correlações que o construto NFA possui com os cinco fatores de personalidade, valores humanos, necessidade de pertença e solidão. Nessa direção, os estudos empíricos serão descritos a seguir.

\section{Método}

Estudo 1. Adaptação da Versão Reduzida do Questionário de Necessidade de Emoções (NAQ-S)

\section{Participantes}

Participaram deste estudo 225 pessoas com idade média de 23,3 anos $(D P=5,69)$, a maioria do sexo feminino $(65,3 \%)$, solteira $(84 \%)$, com ensino superior incompleto $(55,6 \%)$ e se autodeclarando de classe média $(84,4 \%)$. Tratou-se de amostra de conveniência.

\section{Instrumentos}

Além de perguntas de caráter demográfico (sexo, idade, estado civil, nível de escolaridade e classe socioeconômica autopercebida), disponibilizadas ao final, os participantes responderam os seguintes instrumentos:

Questionário de Necessidade de Emoções (NAQ-S). Essa versão foi proposta por Appel et al. (2012), reunindo dez itens distribuídos equitativamente nos fatores Aproximação (e.g., Sinto que necessito vivenciar fortes emocões regularmente; Acho importante explorar meus sentimentos) e Evitação (e.g., Não sei como lidar com minhas emoções, então as evito; Acho as fortes emoções estressantes, portanto, tento evitá-las), respondidos em escala de 7 pontos, variando de -3 (Discordo fortemente) a 3 (Concordo fortemente). Para a tradução dos itens do instrumento, utilizou-se o método back translation. Os itens foram traduzidos para o português por dois pesquisadores bilíngues e, em seguida, retraduzido por outro. Após averiguação das traduções, chegou-se à versão final.

Questionário dos Valores Básicos (QVB). Compreende 18 itens ou valores específicos, que se distribuem em seis fatores ou subfunções valorativas: experimentação, realização, suprapessoal, existência, interativa e normativa. Os itens apresentam descrições breves (e.g., Emoção. Desfrutar desafiando o perigo; buscar aventuras; Afetividade. Ter uma relação de afeto profunda e duradoura; ter alguém para compartilhar seus êxitos e fracassos), onde o participante deve indicar o quão importante (1 - Totalmente não importante a 7 - Extremamente importante) os valores são como um princípio que guia a sua vida (Gouveia, Milfont, Fischer, \& Santos, 2008).

Escala de Solidão de de Jong Gierveld (versão reduzida). Elaborada originalmente por Gierveld e Tilburg (2006), reúne seis itens que avaliam duas dimensões da solidão: Emocional (e.g., Geralmente tenho uma sensação de vazio; Sinto-me rejeitado frequentemente) e Social (e.g., Há muitas pessoas em quem posso confiar completamente; Há muitas pessoas com quem eu posso contar quando tenho problemas), sendo respondidos em escala de cinco pontos (1 = Discordo fortemente a $5=$ Concordo fortemente) .

Escala de Necessidade de Pertença. Elaborada por Leary, Kelly, Cottrell e Schreindorfer (2013), avalia a necessidade de as pessoas pertencerem a determinado grupo. Compreende uma medida unifatorial, reunindo sete itens na versão brasileira (e.g., Não gosto de estar sozinho; Quero que outras pessoas me aceitem), os quais são respondidos em escala de cinco pontos, variando de 1 (Nada) a 5 (Extremamente).

\section{Procedimento}

Realizaram-se coletas on-line e presencial. No primeiro caso, disponibilizou-se um link, onde os partícipes eram informados sobre os objetivos do estudo, indicando que a participação seria voluntária e assegurada a confidencialidade. A coleta presencial foi feita com estudantes universitários, de forma individual, em ambiente coletivo de sala de aula. Nos dois casos, os participantes tiveram que ler e concordar (ou assinar) com um Termo de Consentimento Livre e Esclarecido. Nele se indicava, ademais dos objetivos do estudo, a possibilidade de encerrar antecipadamente a participação sem qualquer ônus e os aspectos éticos da pesquisa. Dois pesquisadores estiveram presentes em sala de aula para dirimir quaisquer dúvidas eventuais dos participantes. Em média, cerca de 20 minutos foram suficientes para concluir sua participação.

\section{Análise de Dados}

Os dados foram analisados com o PASW (versão 22). Calcularam-se estatísticas descritivas (caracterização da amostra), MANOVA (conhecer a qualidade métrica dos itens), análise de componentes principais (checar a estrutura fatorial da medida) e correlação $r$ de Pearson (conhecer a associação da NAQ-S com os valores humanos, solidão e pertença) e análise de confiabilidade. 


\section{Resultados}

Tendo em conta a estrutura fatorial original, computou-se a pontuação para cada fator, criandose, a partir da mediana empírica, os grupos inferior e superior. Posteriormente, calculou-se uma MANOVA entrando com os itens dos fatores respectivos como variáveis dependentes e a classificação dos grupos (inferior e superior) como variável critério. Os itens dos fatores de aproximação lambda de Wilks $=0,36 ; F(5$, $218)=77,27, p<0,001]$ e evitação [lambda de Wilks $=0,34 ; F(5,219)=84,80, p<0,001]$ discriminaram satisfatoriamente os grupos. Aos interessados, a tabela completa com o poder discriminativo dos itens poderá ser disponibilizada por meio de contato com os autores deste artigo.

Assegurada a qualidade métrica dos itens, realizouse uma análise de componentes principais, onde o KMO
$(0,79)$ e o Teste de Esfericidade de Bartlett $\left[\chi^{2}(45)=\right.$ $493,60 ; p<0,001)$ apoiaram que se prosseguisse com esse tratamento multivariado. Não se fixou o número de fatores a extrair, empregando-se rotação varimax. $\mathrm{O}$ critério de Kaiser indicou a extração de dois fatores, com autovalores de 3,08 e 1,89, explicando conjuntamente $49,7 \%$ da variância total. Na Tabela 1 , é possível verificar detalhadamente a estrutura fatorial resultante.

De acordo com a Tabela 1 , o primeiro fator incluiu os itens relativos à Evitação, tendo suas cargas fatoriais variado de 0,60 (Item 4. Acho as fortes emoções estressantes, portanto, tento evitá-las) a 0,77 (Item 10. Emoções são perigosas - elas tendem a me colocar em situacõos que eu preferiria evitar). Sua consistência interna (alfa de Cronbach, $\alpha$ ) foi 0,75 , apresentando homogeneidade (correlação média inter-item) de 0,38 , variando de 0,28 a 0,52 . O segundo fator reuniu os itens de aproximação, apresentando saturações entre 0,61 (Item 9. Para mim é importante saber como

Tabela 1

Estrutura Fatorial da NAQ-S

\begin{tabular}{|c|c|c|c|}
\hline \multirow{2}{*}{ Itens } & \multicolumn{3}{|c|}{ Cargas Fatoriais } \\
\hline & EV & $\mathrm{AP}$ & $h^{2}$ \\
\hline $\begin{array}{l}\text { 10. Emoções são perigosas - elas tendem a me colocar em situações que eu } \\
\text { preferiria evitar. }\end{array}$ & $0,77^{*}$ & $-0,07$ & 0,60 \\
\hline 07. Não sei como lidar com minhas emoções, então as evito. & $0,75^{*}$ & $-0,19$ & 0,60 \\
\hline 06. Preferiria não sentir os altos e baixos das emoções. & $0,70^{*}$ & $-0,03$ & 0,49 \\
\hline $\begin{array}{l}\text { 01. Se eu reflito sobre o meu passado, eu percebo que tendo a ter medo de } \\
\text { sentir emoções. }\end{array}$ & $0,68^{*}$ & 0,09 & 0,47 \\
\hline 04. Acho as fortes emoções estressantes, portanto, tento evitá-las. & $0,60^{*}$ & $-0,18$ & 0,40 \\
\hline 08. Para mim é importante estar em contato com meus sentimentos & $-0,32$ & $0,73^{*}$ & 0,63 \\
\hline 05. Acho importante explorar meus sentimentos. & $-0,21$ & $0,67^{*}$ & 0,50 \\
\hline 02. Sinto que necessito vivenciar fortes emoções regularmente. & 0,18 & $0,66^{*}$ & 0,47 \\
\hline 03. Emoções ajudam a pessoa a se dar bem na vida. & $-0,04$ & $0,66^{*}$ & 0,44 \\
\hline 09. Para mim é importante saber como os outros estão se sentindo. & $-0,05$ & $0,61 *$ & 0,38 \\
\hline Número de itens & 5 & 5 & \\
\hline Valores próprios & 3,08 & 1,89 & \\
\hline Variância explicada & $30,84 \%$ & $18,92 \%$ & \\
\hline Alfa de Cronbach & 0,75 & 0,70 & \\
\hline Correlação inter-item & 0,38 & 0,32 & \\
\hline
\end{tabular}


os outros estão se sentindo) e 0,73 (Item 8. Para mim é importante estar em contato com meus sentimentos). Este apresentou alfa de Cronbach de 0,70 e homogeneidade de 0,32, variando de 0,19 a 0,51 .

Posteriormente, checou-se em que medida as pontuações dos fatores da NAQ-S se correlacionavam com valores humanos, necessidade de pertença e solidão, construtos estes que podem ser úteis para o entendimento dos fatores Aproximação e Evitação, aportando evidências complementares de validade convergente desse questionário. Os resultados a respeito são apresentados na Tabela 2 a seguir.

Tabela 2

Correlatos da Necessidade de Emocões

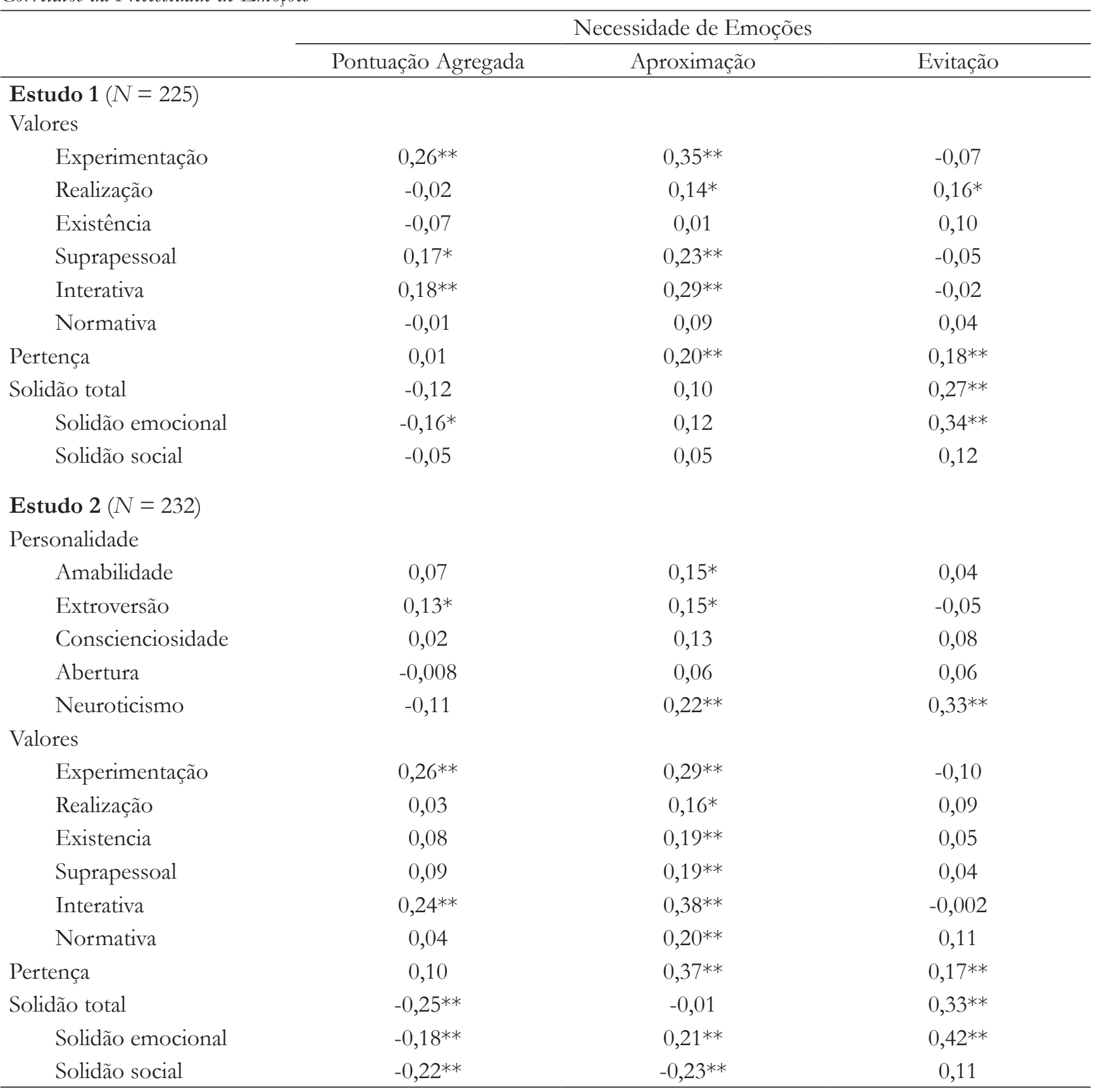

Nota. $* * p<0,01 ; * p<0,05$. 
Considerando a pontuação agregada da necessidade de emoções (Aproximação - Evitação), verificou-se que esta se correlacionou com três subfunções valorativas: Experimentação $(r=0,26, p<0,01)$, Interativa $(r$ $=0,18, p<0,01)$ e Suprapessoal $(r=0,17, p<0,05)$. Também se observou correlação entre a necessidade de emoções e a Solidão emocional $(r=-0,16, p<0,05)$.

Por fim, quanto aos fatores específicos da necessidade de emoções, observaram-se algumas variações nos coeficientes de correlação. Concretamente, o fator Aproximação se correlacionou com as subfunções valorativas Experimentação $(r=0,35, p<0,01)$, Interativa $(r=0,29, p<0,01)$, Suprapessoal $(r=0,23, p<$ $0,01)$ e Realização $(r=0,14, p<0,05)$, ao passo que Evitação o fez apenas com realização $(r=0,16, p<$ $0,05)$. Quanto às correlações com Necessidade de pertença, estas foram evidentes tanto para Aproximação $(r$ $=0,20, p<0,01)$ como Evitação $(r=0,18, p<0,05)$. Por fim, Evitação se correlacionou com a pontuação total de Solidão $(r=0,27, p<0,01)$ e o fator Solidão emocional $(r=0,34, p<0,01)$.

Estudo 2. Comprovação do Modelo e Invariância Fatorial da NAQ-S

\section{Participantes}

Participaram deste estudo 232 pessoas da população geral com idade média de 26,3 anos $(D P=7,06)$, a maioria do sexo feminino $(54,3 \%)$, indicando ter ensino superior incompleto $(40,5 \%)$ e se autopercebendo como de classe socioeconômica média $(83,5 \%)$. Tratou-se de amostra de conveniência.

\section{Instrumentos}

Além de serem aplicados os mesmos instrumentos do Estudo 1, os participantes responderam o Inventário dos Cinco Grandes Fatores (ICGF). Essa medida foi proposta por John, Donahue e Kentle (1991), composta por 44 itens, sendo adaptado para o Brasil por Andrade (2008). Não obstante, seguindo a linha de primar por medidas curtas, optou-se por sua versão reduzida, composta por 20 itens, sendo os quatro com maiores cargas fatoriais de cada fator, segundo descrito no estudo de Schmitt, Allik, McCrae e Benet-Martínez (2007). Os itens são estruturados em sentenças (e.g., É sociável, extrovertido; É amável, tem consideração pelos outros), sendo o indivíduo solicitado a indicar como se percebe (Eu me vejo como alguém que...), utilizando escala de resposta de cinco pontos, variando de 1 (Discordo totalmente) a 5 (Concordo totalmente).

\section{Procedimento}

Os dados foram coletados exclusivamente on-line, sendo o link da pesquisa divulgado por meio das redes sociais. Os participantes foram informados que sua participação seria voluntária, preservando-se seu anonimato e o sigilo de suas respostas. Antes de prosseguirem, deveriam ainda concordar com um Termo de Consentimento Livre e Esclarecido, contendo informações sobre o estudo e o seu caráter ético. Aqueles que o fizeram levaram, em média, 20 minutos para concluir sua participação.

\section{Análise de Dados}

Os dados foram analisados com os pacotes estatísticos PASW e AMOS (versões 22). Com o primeiro foram calculadas as estatísticas descritivas e correlação $r$ de Pearson. Em se tratando do segundo programa, ele foi utilizado para comprovar a estrutura bifatorial da NAQ-S e checar sua invariância fatorial quanto ao sexo dos participantes.

\section{Resultados}

Inicialmente, testou-se o modelo bifatorial identificado no Estudo 1, conforme se descreve na Figura 1, sendo seus indicadores de ajuste aceitáveis: $\chi^{2} / g l=2,67$, GFI $=0,92$, AGFI $=0,88$, TLI $=0,86$, CFI $=0,90 \mathrm{e}$ RMSEA $=0,085(I C 90 \%=0,064-0,107)$. Destaca-se que todos os lambdas foram estatisticamente diferentes de zero $(z \neq 0 ; z>1,96, p<0,05)$, variando de 0,55 (Item 6. Preferiria não sentir os altos e baixos das emoçoes) a 0,74 (Item 7. Não sei como lidar com minhas emoçoes, então as evito) para o fator Evitação e de 0,36 (Item 9. Para mim é importante saber como os outros estão se sentindo) a 0,81 (Item 5. Acho importante explorar meus sentimentos) para Aproximação.

Posteriormente, realizou-se uma análise fatorial confirmatória multigrupo, visando conhecer se a NAQ-S é invariante quanto ao sexo dos participantes. Tradicionalmente, para se checar essa propriedade da medida, tem se recomendado levar em conta o $\Delta \chi^{2}$. Porém, esse índice apresenta algumas limitações no que diz respeito à sensibilidade ao tamanho amostral (Damásio, 2013). Nessa direção, além do $\Delta \chi^{2}$, levou-se em conta o $\triangle$ RMSEA, onde a comparação entre o valor do modelo avaliado e do prévio não deve exceder 0,015 (Wu, Li, \& Zumbo, 2007). Os resultados dessa análise podem ser observados na Tabela 3.

Percebe-se nessa tabela que o $\Delta \chi^{2}$ apoia as invariâncias configural e métrica, contudo, não dá 
suporte à estrutural e residual. Nesse caso, decidiu-se calcular as diferenças entre os valores do RMSEA, que, ao longo das restrições impostas, deu suporte a um modelo invariante (Wu et al., 2007).

Posteriormente, buscou-se verificar correlações entre necessidade de emoções e os mesmos construtos do Estudo 1, porém foram incluídos no presente estudo os cinco grandes fatores da personalidade. As correlações a respeito podem ser verificadas na Tabela 2, evidenciando que são similares àquelas do estudo anterior. Especificamente, observou-se que a pontuação total da NAQ-S se correlacionou com as subfunções valorativas Experimentação $(r=0,26, p<0,01)$ e Interativa $(r=0,24, p<0,01)$. Ademais, a necessidade de emoções se correlacionou negativamente com a pontuação total da medida de solidão $(r=-0,25, p<0,01)$, assim como com seus dois fatores: Social $(r=-0,22, p$ $<0,01)$ e Emocional $(r=-0,18, p<0,01)$. Com relação aos traços de personalidade, a pontuação agregada da necessidade de emoções se correlacionou unicamente com Extroversão $(r=0,13, p<0,05)$.

Considerando os fatores específicos da NAQ$S$, verificou-se que todas as subfunções valorativas se correlacionaram com Aproximação, sendo as correlações mais fortes com Experimentação $(r=0,29, p<$ $0,01)$ e Interativa $(r=0,38, p<0,01)$. Os dois fatores de solidão também se correlacionaram com o fator Aproximação, e que este fator e Evitação se correlacionaram com necessidade de pertença. Por fim, Aproximação se correlacionou com Neuroticismo $(r=0,22, p<0,01)$, Amabilidade $(r=0,15, p<0,05)$ e Extroversão $(r=$

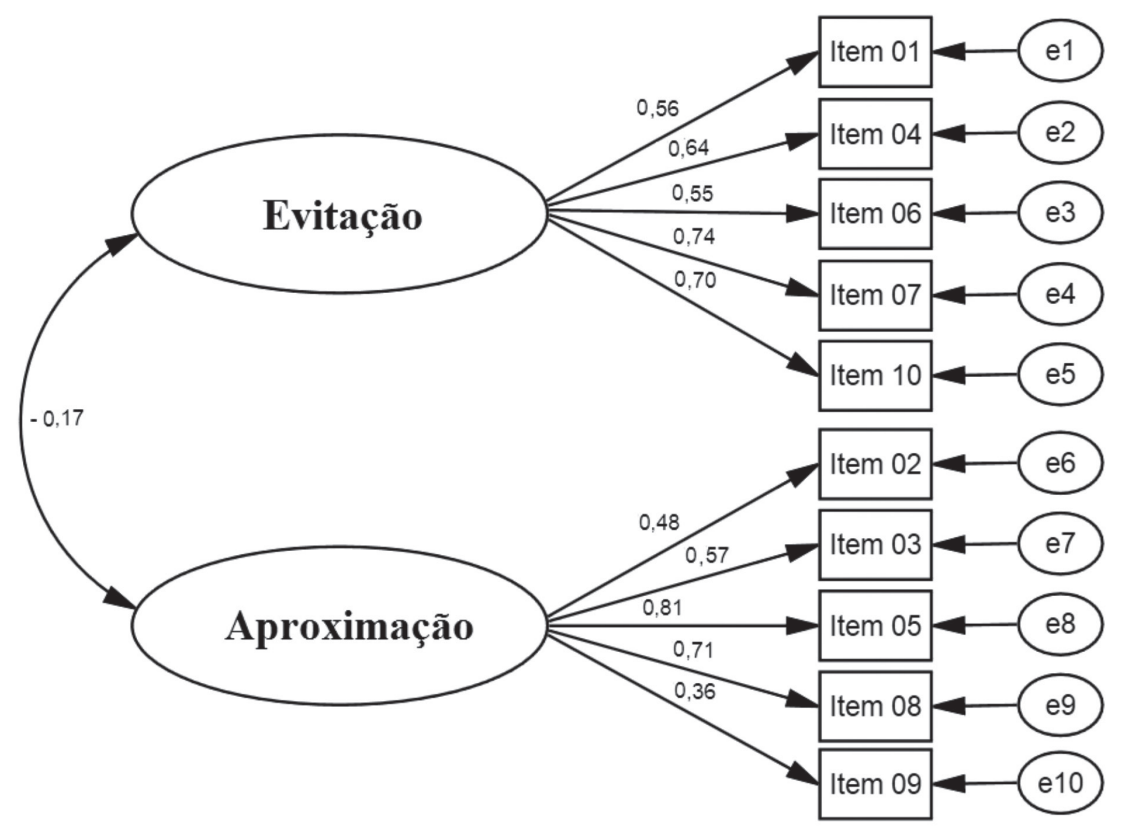

Figura 1. Estrutura bifatorial da NAQ-S.

Tabela 3

Invariância Fatorial da NAQ-S quanto ao Sexo

\begin{tabular}{lcccc}
\hline Modelos & $\chi^{2}(g)$ & $\Delta \chi^{2}(g D$ & RMSEA & $\Delta$ RMSEA \\
\hline Invariância Configural & $129,738(68)$ & - & 0,063 & - \\
Invariância Métrica & $137,509(76)$ & $7,771(8)$ & 0,059 & 0,004 \\
Invariância Estrutural & $146,975(79)$ & $9,466(3)^{*}$ & 0,061 & 0,002 \\
Invariância Residual & $166,10(89)$ & $19,462(10)^{*}$ & 0,062 & 0,001 \\
\hline
\end{tabular}

Nota. ${ }^{*} p<0,05$ 
0,15, $p<0,05)$, enquanto que Evitação o fez apenas com Neuroticismo $(r=0,33, p<0,01)$.

\section{Discussão}

Considerando o número reduzido de estudos sobre a necessidade de emoções, não sendo encontrada qualquer pesquisa a respeito no contexto brasileiro, decidiu-se empreender esforços para suprir esta lacuna, procurando incialmente adaptar a versão reduzida do Questionário de Necessidade de Emoções (NAQ-S), reunindo evidências de validade (fatorial e convergente) e consistência interna. Estima-se que esses objetivos tenham sido alcançados, embora se reconheçam limitações potenciais dos estudos. Especificamente, destaca-se que as amostras não foram probabilísticas, restringindo a generalização dos resultados. Outra potencial limitação reside na desejabilidade social ou viés de resposta associado à medida de autorrelato.

Tais limitações não invalidam os achados antes reportados, que foram na direção do que tem sido observado em outros contextos. No que concerne aos parâmetros psicométricos, verificou-se no Estudo 1 que dois fatores foram encontrados, coerente com o que observaram Maio e Esses (2001) com a versão original, e Appel et al. (2012) com sua versão reduzida. Destaca-se que as cargas fatoriais foram acima do recomendado pela literatura e que os indicadores de consistência interna (alfa de Cronbach) e homogeneidade (correlação média inter-item) foram aceitáveis (Clark \& Watson, 1995; Pasquali, 2010).

A estrutura bifatorial teorizada foi corroborada no Estudo 2. Os indicadores de ajuste desse modelo foram na direção do que se preconiza na literatura (Byrne, 2013; Hair, Black, Babin, \& Anderson, 2015). Os resultados oriundos dos dois estudos endossam a estrutura de dois fatores, dando suporte a se pensar a NFA como uma atitude frente às emoções, havendo atitudes positivas e negativas (Maio \& Esses, 2001). Ademais, é possível observar que estudos em diferentes culturas (e.g., Reino Unido, Itália e Alemanha; Appel, 2008; Appel et al., 2012; Leone \& Chirumbolo, 2008) vêm chegando a essa mesma conclusão, reforçando que as motivações para se aproximar ou não das emoções é uma necessidade pancultural (Maio \& Esses, 2001).

Em relação à invariância fatorial, decidiu-se testá-la tendo em conta o sexo dos participantes, que é uma variável relevante para explicar a variabilidade na necessidade de emoções (Appel et al., 2012). Concretamente, como indicam Maio e Esses (2001), homens podem aprender a evitar emoções, pois expressá-las seria algo incongruente com as concepções de masculinidade, sendo considerado um sinal de fraqueza (Guerra, Scarpati, Brasil, Livramento, \& Silva, 2015). Por outro lado, a figura tradicional das mulheres como cuidadoras pode levá-las a se aproximarem de emoções, permitindo-lhes a identificação com as experiências emocionais dos outros. Desse modo, conhecer a invariância da medida é relevante, pois eventuais diferenças podem refletir a influência do sexo, e não o fato de a medida não funcionar adequadamente em homens e mulheres. No caso, observou-se, no Estudo 2, evidências de um funcionamento adequado da NAQ-S para homens e mulheres, onde o cálculo das diferenças entre os RMSEAs dos modelos apontou invariância (Wu et al., 2007).

No que tange às correlações da necessidade de emoções com traços de personalidade, observou-se que sua pontuação agregada se correlacionou apenas com o traço extroversão. Considerando especificamente os fatores da NAQ-S, verificou-se que Aproximação se correlacionou com Amabilidade e Extroversão, sugerindo o perfil de alguém que busca o contato social, sendo que as emoções inerentes ao envolvimento com outras pessoas são elementos que podem auxiliar no entendimento de tais correlações (Appel et al., 2012; Maio \& Esses, 2001). O traço Neuroticismo se correlacionou significativamente com os fatores de Aproximação e Evitação, como visto em outros estudos (Appel et al., 2012; Maio \& Esses, 2001). O traço caracteriza indivíduos que são emocionalmente instáveis, tendo dificuldades em controlar as emoções vivenciadas e ficando tensos e nervosos facilmente. Sendo assim, considera-se plausível a correlação com os dois fatores, uma vez que esses indivíduos vivenciam intensamente as emoções (Aproximação), de forma descontrolada, o que poderia levá-los a evitar tais situações (Evitação).

Considerando as correlações com valores humanos, percebe-se que nos dois estudos a pontuação total da necessidade de emoções se correlacionou positivamente com as subfunções experimentação e interativa. Especificamente, os valores de experimentação e seus marcadores (Sexualidade, Emoção e Prazer) descrevem pessoas pautadas na busca de sensação, com um perfil hedônico, abertas a novas experiências (Gouveia et al., 2014). Portanto, assumindo os valores como expressão das necessidades, pode-se pensar que a priorização de valores dessa subfunção pode ser respostas à necessidade de se aproximar de situações que possam eliciar emoções intensas. Por outro lado, sua correlação com os valores da subfunção interativa demarcam pessoas 
que valorizam o contato interpessoal, sendo as relações um fim em si mesma, onde são enfatizados atributos mais afetivos (Gouveia, 2013; Gouveia et al., 2014). Logo, a busca por tais relações e as emoções que são oriundas delas podem explicar tais correlações (Appel et al., 2012; Maio \& Esses, 2001). Nessa direção, como pontuam Maio e Olson (1998), os valores podem ser influenciados pelas emoções, sobretudo aqueles sociais; segundo Gouveia (2013), os humanitários, como das subfunções Experimentação e Interativa também potencializam a abertura a emoções.

Verificou-se, ainda, que a necessidade de emoções se correlacionou com dois construtos estudados no campo dos relacionamentos interpessoais: necessidade de pertença e solidão. Com o primeiro, reforça-se a ideia de que pertencer a um grupo social e as emoções que derivam dessas relações pode instigar a aproximação de emoções, vivenciando-as. Por outro lado, como verificado nos dois estudos, a necessidade parece incompatível com o sentimento de solidão, indicando que pessoas que o vivenciam evitam entrar em contato com suas emoções e aquelas dos demais ao seu redor (Maio \& Esses, 2001).

Por fim, apesar dos resultados reportados serem coerentes com a literatura, percebem-se possibilidades de estudos futuros que reúnam evidências que endossem a adequação da NAQ-S, favorecendo fortalecer pesquisas sobre as necessidades de emoções. De fato, foram encontrados poucos estudos a respeito, sendo que nenhum, no Brasil. Como possibilidades futuras, é importante contar com amostras mais heterogêneas, assim como controlar o viés da desejabilidade social. Ademais, será importante conhecer o impacto da necessidade em emoções sociais (e.g., culpa, vergonha), além de conhecer a estabilidade temporal (teste-reteste) da NAQ-S ou explorar os seus parâmetros psicométricos por meio da Teoria de Resposta ao Item.

\section{Referências}

Andrade, J. M. (2008). Evidências de validade do Inventário dos cinco grandes fatores de personalidade para o Brasil (Tese de doutorado). Departamento de Psicologia, Universidade de Brasília, DF.

Appel, M. (2008). Manche mögen's heiß. Diagnostica, 54, 2-15. doi: 10.1026/0012-1924.54.1.2

Appel, M., Gnambs, T., \& Maio, G. R. (2012). A short measure of the need for affect. Journal of Personality Assessment, 94, 418-426. doi: 10.1080/00223891.2012.666921
Arceneaux, K., \& Wielen, R. J. V. (2013). The effects of need for cognition and need for affect on partisan evaluations. Political Psychology, 34, doi: 10.1111/j.1467-9221.2012.00925.x

Baumeister, R. F., \& Leary, M. R. (1995). The need to belong: Desire for interpersonal attachments as a fundamental human motivation. PsychologicalBulletin, 117, 497-529. doi: 10.1037/0033-2909.117.3.497

Byrne, B. M. (2010). Structural Equation Modeling with AMOS: Basic Concepts, Applications, and Programming. New York: Routledge.

Cho, S., \& Workman, J. E. (2014). Relationships among gender, fashion leadership, need for affect, and consumers' apparel shopping preference. Family \& Consumer Sciences, 42, 369-385. doi: 10.1111/ fcsr. 12070

Clark, L. A., \& Watson, D. (1995). Constructing validity: Basic issues in objective scale development. Psychological Assessment, 7, 309-319. doi: 10.1037/1040-3590.7.3.309

Corwin, E. P., Cramer, R. J., Griffin, D. A., \& Brodsky, S. L. (2012). Defendant remorse, need for affect, and juror sentencing decisions. The Journal of the American Academy of Psychiatry and the Law, 40, 41-49. Recuperado de http://www.jaapl.org/ content/40/1/41.long

Dalgleish, T., Dunn, B. D., \& Mobbs, D. (2009). Affective neuroscience: Past, present, and future. Emotion Review, 1, 355-368. doi: 10.1177/1754073909338307

Damásio, B. F. (2013). Contribuições da análise fatorial confirmatória multigrupo (AFCMG) na avaliação de invariância de instrumentos psicométricos. Psico-USF, 18, 211-220. doi: 10.1590/ S1413-82712013000200005

Gierveld, J. D. J., \& Tilburg, T. V. (2006). A 6-Item scale for overall, emotional, and social loneliness confirmatory tests on survey data. Research on Aging, 28, 582-598. doi: 10.1177/0164027506289723

Gosling, S. D., Rentfrow, P. J., \& Swann, W. B. (2003). A very brief measure of the Big-Five personality domains. Journal of Research in Personality, 37, 504-528. doi: 10.1016/S0092-6566(03)00046-1

Gouveia, V. V. (2013). Teoria funcionalista dos valores humanos: Fundamentos, aplicações e perspectivas. São Paulo: Casa do Psicólogo. 
Gouveia, V. V., Milfont, T. L., \& Guerra, V. M. (2014). Functional theory of human values: Testing its content and structure hypotheses. Personality and Individual Differences, 60, 41-47. doi: 10.1016/j. paid.2013.12.012

Gouveia, V. V., Milfont, T. L., Fischer, R. \& Coelho, J. A. P. M. (2009). Teoria funcionalista dos valores humanos: aplicações para organizações. Em M. L. M. Teixeira (Org.), Valores Humanos \& Gestão: Novas perspectivas (pp.47-80). São Paulo: Senac.

Guerra, V. M., Scarpati, A. S., Brasil, J. A., Livramento, A. M., \& Silva, C. V. (2015). Concepções de masculinidade: Suas associações com os valores e a honra. Psicologia e Saber Social, 4, 72-88. doi: 10.12957/psi. saber.soc.2015.14840

Haddock, G., Maior, G. R., Arnold, K., \& Huskinson, T. (2008). Should persuasion be affective or cognitive? The moderating effects of need for affect and need for cognition. Personality and Social Psychology Bulletin, 34, 769-778. doi: 10.1177/0146167208314871

Hair, J. F. J., Black, W. C., Babin, B. J., \& Anderson, R. E. (2015). Multivariate Data Analysis ( $7^{\mathrm{a}}$ Ed.). Upper Saddle River, NJ: Prentice Hall.

John, O. P., Donahue, E. M., \& Kentle, R. L. (1991). The "Big Five" Inventory \& Versions $4 a$ and 54. Berkeley, University of California. Institute of Personality and Social Research.

Leary, M. R., Kelly, K. M., Cottrell, C. A., \& Schreindorfer, L. S. (2013). Construct validity of the need to belong scale: Mapping the nomological network. Journal of Personality Assessment, 95, 610-624. doi: 10.1080/00223891.2013.819511

Leone, L., \& Chirumbolo, A. (2008). Conservatism as motivated avoidance of affect: Need for affect scales predict conservatism measures. Journal of Research in Personality, 42, 755-762. doi: 10.1016/j. jrp.2007.08.001

Leone, L., \& Presaghi, F. (2007). Validity of the need for affect scales: Factorial structure, invariance and validity in Italy. TPM-Testing, Psychometrics, Methodology in Applied Psychology, 14, 117-134. doi: 10.4473/ TPM.14.3-4.1

Lutz, C., \& White, G. M. (1986). The anthropology of emotions. Annual Review of Antropology, 15, 405-436. Recuperado de http://www.jstor.org/ stable/2155767
Maio, G. R., \& Esses, V. M. (2001). The need for affect: Individual differences in the motivation to approach or avoid emotions. Journal of personality, 69, 583-614. doi: 10.1111/1467-6494.694156

Maio, G. R., Esses, V. M., Arnold, K., \& Olson, J. M. (2004). The function-structure model of attitudes: Incorporating the need for affect. Em Contemporary perspectives on the psychology of attitudes (pp. 9-34). Recuperado de http://dialnet.unirioja.es/servlet/ articulo? codigo $=4607684$

Maio, G. R., \& Olson, J. M. (1998). Values as truisms: Evidence and implications. Journal of Personality and Social Psychology, 74, 294-311. doi: 10.1037/0022-3514.74.2.294

Mellor, D., Stokes, M., Firth, L., Hayashi, Y., Cummins, R. (2008). Need for belonging, relationship satisfaction, loneliness, and life satisfaction. Personality and Individual Differences, 45, 213-218. doi: 10.1016/j.paid.2008.03.020

Pasquali, L. (2010). Instrumentação Psicológica: Fundamentos e práticas. Artmed.

Raman, N. V., Chattopadhyay, P., \& Hoyer, W. D. (1995). Do consumers seek emotional situations: The need for emotion scale. Advances in Consumer Research, 22, 537-542. Recuperado de http://acrwebsite.org/ volumes/7804/volumes/v22/NA-22

Rammstedt, B., \& John, O. P. (2007). Measuring personality in one minute or less: A 10-item short version of the Big Five Inventory in English and German. Journal of Research in Personality, 41, 203212. doi: $10.1016 /$ j.jpr.2006.02.001

Schmitt, D. P., Allik, J., McCrae, R. R., \& Benet-Martínez, V. (2007). The geographic distribution of big five personality traits patterns and profiles of human self-description across 56 nations. Journal of Cross-Cultural Psychology, 38, 173-212. doi: $10.1177 / 0022022106297299$

Wu, A. D., Li, Z., \& Zumbo, B. D. (2007). Decoding the meaning of factorial invariance and updating the practice of multi-group confirmatory factor analysis: A demonstration with TIMSS Data. Practical Assessment, Research \& Evaluation, 12, 1-26. Recuperado de http://pareonline.net/getvn. asp?v $=12 \& n=3$

Recebido em: 23/02/2016

Reformulado em: 06/05/2016

Aprovado em: 24/05/2016 
Nota dos autores:

Agradecimentos: Coordenação de Aperfeiçoamento de Pessoal de Nível Superior (CAPES) pelas bolsas de doutorado concedidas ao primeiro e último autor.

Sobre os autores:

Gabriel Lins de Holanda Coelho possui formação em Psicologia e mestrado em Psicologia Social pela Universidade Federal da Paraíba. Atualmente é doutorando em Psicologia pela Cardiff University, País de Gales. Seus interesses de pesquisa centram-se na psicologia social dos valores humanos, relacionamentos interpessoais e construção e adaptação de medidas.

E-mail:linshc@gmail.com

Gregory R. Maio é professor e chefe do departamento de Psicologia da University of Bath, Inglaterra. É autor de diversas publicações, como os livros "The psychology of attitudes and attitude change", "Attitudes" e "The psychology of buman values". Seus interesses de pesquisa centram-se na psicologia social dos valores humanos, atitudes e processos emocionais.

E-mail: g.r.maio@bath.ac.uk

Valdiney V. Gouveia é professor titular de Psicologia Social na Universidade Federal da Paraíba (http://vvgouveia. net) e pesquisador Nível 1A do CNPq, atuando como consultor de agências nacionais e internacionais de fomento à pesquisa. É autor de diversas publicações, como o livro "Teoria Funcionalista dos Valores Humanos: Fundamentos, Aplicações e Perspectivas", teoria a qual desenvolveu e tem dedicado-se em suas pesquisas.

E-mail:vvgouveia@gmail.com

Lukas Jarmo Wolf é mestre em Ciência do Comportamento na Radboud University Nijmegen, Holanda, e doutor em Psicologia na Cardiff University, País de Gales. Atualmente é pesquisador assistente na Cardiff University. Seus interesses de pesquisa centram-se em formação de estereótipos e preconceito, relacionamentos interpessoais, psicologia social dos valores humanos e percepção intergrupal.

E-mail:1.wolf@bath.ac.uk

Renan Pereira Monteiro possui formação em Psicologia pela Universidade Federal do Piauí, mestrado e doutorado em Psicologia Social pela Universidade Federal da Paraíba. Atualmente é professor do departamento de Psicologia da Universidade Federal de Mato Grosso (Campus Cuiabá). Seus interesses de pesquisa centram-se no lado sombrio da personalidade, psicologia social dos valores humanos, construção e adaptação de medidas psicológicas.

E-mail: renanpmonteiro@gmail.com

Contato com os autores:

Gabriel Coelho

School of Psychology, Tower Building

70 Park Place, Cardiff (United Kingdom)

CF10 3AT. 